\title{
Metal-insulator transition induced by random dipoles
}

\author{
M. Larcher, ${ }^{1}$ C. Menotti, ${ }^{1}$ B. Tanatar, ${ }^{2}$ and P. Vignolo ${ }^{3}$ \\ ${ }^{1}$ INO-CNR BEC Center and Dipartimento di Fisica, Università di Trento, 38123 Povo, Italy \\ ${ }^{2}$ Department of Physics, Bilkent University, Bilkent, 06800 Ankara, Turkey \\ ${ }^{3}$ Université de Nice - Sophia Antipolis, Institut non Linéaire de Nice, CNRS, 1361 route des Lucioles, 06560 Valbonne, France
}

(Received 22 March 2013; published 26 July 2013)

\begin{abstract}
We study the localization properties of a test dipole feeling the disordered potential induced by dipolar impurities trapped at random positions in an optical lattice. This random potential is marked by correlations which are a convolution of short-range and long-range ones. We show that when short-range correlations are dominant, extended states can appear in the spectrum. Introducing long-range correlations, the extended states, if any, are wiped out and localization is restored over the whole spectrum. Moreover, long-range correlations can either increase or decrease the localization length at the center of the band, which indicates a richer behavior than previously predicted.
\end{abstract}

DOI: 10.1103/PhysRevA.88.013632

PACS number(s): 03.75.-b, 64.60.Cn, 71.23.An

\section{INTRODUCTION}

Interference effects induced by random potentials deeply modify wave diffusion up to even stopping wave transport on a length $\mathcal{L}_{\text {loc }}$, the localization length. This phenomenon called strong localization or Anderson localization [1,2] can be observed in classical waves such as acoustic waves [3], microwaves [4], light [5], as well as in quantum waves, such as electronic [6] or matter waves in real [7-10] or momentum space $[11,12]$. Anderson theory relies essentially on two main assumptions: (i) the potential has to be $\delta$ correlated; (ii) the wave has to be noninteracting. If these conditions are fulfilled, Anderson localization occurs always in one and two dimensions, and depending on the disorder strength and the energy, in three dimensions [13]. A localization-delocalization transition can appear in low dimensions if correlations and/or interactions are introduced in the systems. If on the one hand noninteracting waves exist in nature, on the other hand real uncorrelated potentials do not exist. Correlated disordered potentials can be roughly divided into three wide classes, according to the behavior of the two-point autocorrelation function $C(\ell)$ and of its Fourier transform $S(k)$. The first class is marked by a correlation function $C(\ell)$ which decays exponentially on a length $\bar{\ell}$ with $\pi / k_{\max }<\bar{\ell} \ll L, L$ being the length of the system and $k_{\max }$ the largest wave vector allowed by the system. These potentials, called short-range correlated potentials, may introduce resonance energies in the spectrum inducing delocalization of a significant subset of the eigenstates. This happens for instance in the random-dimer model (RDM) and in its dual counterpart (DRDM) [14-17], in which the sites of a lattice are assigned energies $\varepsilon_{a}$ or $\varepsilon_{b}$ at random, with the additional constraint that sites of energy $\varepsilon_{b}$ always appear in pairs (RDM) or never appear as neighbors (DRDM). The second class of correlated potentials is marked by a spectral function $S(k)$ that is nonzero in a finite $k$ range. This is, for instance, the case of the speckle $[18,19]$ where $C(\ell)$ is a sinc function. For these kinds of potentials, there exists a critical energy at which the localization length increases abruptly mimicking the presence of a mobility edge in finite-size systems [20,21]. In the third class $C(\ell)$ decreases algebraically as $\sim 1 / \ell^{\beta}$, and both $C(\ell)$ and $S(k)$ are nonzero over the whole real and $k$ spaces. In practice there are no length scales characterizing the disorder, which is scale free. These potentials are commonly called long-range correlated. In this case, it has been observed that correlations can have different effects depending on the energy region under consideration. In particular, for discrete models, a reduction of the localization length has been observed at the band edges and conversely an enhancement at the band center has been reported [22]. In this context also the presence of mobility edges has been claimed [23], although these results stirred some controversy [24,25].

Very often, especially in the case of long-range correlations, these studies rely on toy models characterized by $a d-h o c$ correlation functions, creating almost no connection with possible experimental implementations. In this paper we propose a physical model for a random potential where long-range and short-range correlations arise naturally from the system itself, exploiting the properties of the dipolar interaction [26,27] in ultracold atomic [28-31] or molecular $[32,33]$ gases. The model considers a series of dipoles pinned at random positions at the minima of a deep optical lattice. The dipoles are polarized perpendicularly to the lattice axis, so that dipole-dipole interaction is repulsive. In this way, for low enough densities, there are no double occupancies, and, if the dipole-dipole interaction is large enough, consecutive occupations are avoided too. This set of trapped dipoles, referred as impurities, will create a disordered potential $[15,34,35]$ for another dipole, the test dipole, which is excited to a different internal level and is able to move through the lattice (see Fig. 1). Short-range correlations arise from the distribution of the impurities, while long-range correlations are due to the dipolar interaction between the test dipole and the impurities.

We study the localization properties of the test dipole in the correlated potential realized by the impurities, highlighting the role played by short- and long-range correlations. In particular, depending on the parameters of the model, we observe that short-range correlations can introduce a discrete set of extended states in the system while long-range correlations tend to restore localization and lead to counterintuitive effects on the localization length of the system.

The paper is organized as follows. The model is presented in detail in Sec. II and the Hamiltonian for the test dipole is derived. In Sec. III, we study the localization properties 


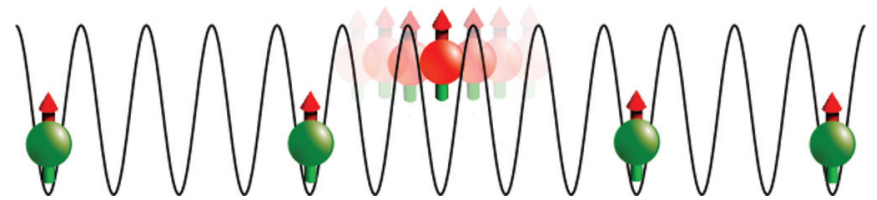

FIG. 1. (Color online) Schematic representation of the physical model. Dipolar impurities (green spheres) are trapped at the minima of the optical lattice, occupying random positions. The test dipole (red upper sphere), excited to another internal level, feels a shallower optical potential and a disordered potential due to the dipolar interaction with the impurities.

of the model by using a renormalization-decimation scheme for the calculation of the localization length. Then, a detailed discussion of the role played by short and long correlations is presented in Sec. IV. Finally, we draw our conclusions in Sec. V.

\section{THE PHYSICAL MODEL}

We consider a very dilute gas of dipolar impurities trapped in a deep one-dimensional (1D) optical lattice and a single test dipole excited to a different internal level with the same dipole moment $\vec{D}$. We superimpose to the optical lattice a very elongated cigar-shaped harmonic confinement, so that we can practically neglect the weak axial harmonic confinement along the $z$ axis. We assume both for the impurities and the test dipoles, frozen radial dynamics into the lowest state of the radial harmonic confinement

$$
\phi_{\omega}\left(\vec{r}_{\perp}\right)=\frac{1}{\sqrt{\pi} \sigma_{\omega}} e^{-r_{\perp}^{2} / 2 \sigma_{\omega}^{2}} \quad \text { with } \quad \sigma_{\omega}=\sqrt{\frac{\hbar}{m \omega}},
$$

where $\omega=\omega^{(I)}$ and $\omega=\omega^{(T)}$ for impurities and the test particle, respectively.

Furthermore, assuming that the optical lattice felt by the impurities is so strong as to completely freeze their dynamics along $z$, we can describe the motion of the test particle of mass $m$ along the lattice axis $z$ by the following 1D effective Hamiltonian

$$
H=-\frac{\hbar^{2}}{2 m} \frac{\partial^{2}}{\partial z^{2}}+s E_{R} \sin ^{2}(k z)+V_{d}(z),
$$

where $E_{R}=\pi^{2} \hbar^{2} / 2 m d_{L}^{2}$ is the recoil energy, $d_{L}$ the lattice spacing of an optical lattice generated by a laser of wavelength $\lambda_{L}=2 d_{L}$, and $s$ the adimensional lattice depth.

The random potential $V_{d}(z)$ results from the dipolar interaction of the test particle with the impurities pinned in the lattice. It is given by the convolution of the density distribution of the impurities $\rho(z)$ [see Eq. (4)] and the effective one-dimensional dipolar interaction $U_{d d}^{1 D}(z)$ [see Eq. (5)]:

$$
V_{d}(z)=\int d z^{\prime} \rho\left(z^{\prime}\right) U_{d d}^{1 D}\left(z-z^{\prime}\right) .
$$

The density distribution $\rho(z)$ is given by the sum of the Wannier functions $w^{(I)}(z)$ localized around the sites $\bar{l}$ occupied by the impurities

$$
\rho(z)=\sum_{\bar{l}}\left|w^{(I)}\left(z-\bar{l} d_{L}\right)\right|^{2}
$$

The random distribution of the occupied sites $\bar{l}$ introduces disorder in the system.

The effective one-dimensional dipolar potential $U_{d d}^{1 D}(z)$ is obtained after integration of the dipolar interaction $U_{d d}(\vec{r})=$ $D^{2}\left[1-3(\hat{D} \cdot \hat{r})^{2}\right] /|\vec{r}|^{3}$ in the radial directions and is given by [36]

$$
\begin{aligned}
U_{d d}^{1 D}(z)= & \int d \overrightarrow{r^{\prime}} \perp d \vec{r}_{\perp}\left|\phi_{\omega^{(I)}}\left(\vec{r}_{\perp}^{\prime}\right)\right|^{2}\left|\phi_{\omega^{(T)}}\left(\vec{r}_{\perp}\right)\right|^{2} U_{d d}\left(\vec{r}-\vec{r}^{\prime}\right) \\
= & \frac{D^{2}}{\sigma_{\perp}^{3}}\left(1-3 \cos ^{2} \alpha\right)\left\{-\frac{2}{3} \delta\left(\frac{z}{\sigma_{\perp}}\right)+\frac{1}{2} \sqrt{\frac{\pi}{2}} e^{\frac{1}{2} \frac{z^{2}}{\sigma_{\perp}^{2}}}\right. \\
& \left.\times\left[\left(\frac{z^{2}}{\sigma_{\perp}^{2}}\right)+1\right] \operatorname{erfc}\left(\frac{|z|}{\sqrt{2} \sigma_{\perp}}\right)-\frac{|z|}{2 \sigma_{\perp}}\right\}
\end{aligned}
$$

where $\sigma_{\perp}=\sqrt{\left(\sigma_{\omega^{(I)}}^{2}+\sigma_{\omega^{(T)}}^{2}\right) / 2}$ is the radial width of the system, $\alpha$ is the angle between the dipole moment $\vec{D}$ and the $z$ axis, and $\operatorname{erfc}(z)$ is the complementary error function

$$
\operatorname{erfc}(z)=\frac{2}{\sqrt{\pi}} \int_{z}^{\infty} e^{-t^{2}} d t
$$

Note that the final expression that we obtained for $U_{d d}^{1 D}(z)$ is composed of two parts: a Dirac $\delta$ term at $z=0$ and a slowly decaying part. One can show that at large distances $|z| \gg \sigma_{\perp}$, the slowly decaying part reproduces the typical behavior of the dipolar interaction, namely, a decrease with the inverse cubic distance $U_{d d}^{1 D}(z) \sim D^{2}\left(1-3 \cos ^{2} \alpha\right) /|z|^{3}$. For simplicity, in the present paper, we do not include contact interactions with the underlying idea that they can be switched off by exploiting Feshbach resonances [37]. However, they would simply modify the strength of the $\delta$ part of the 1D potential and provide an additional way to tune the parameters of the system.

The tight binding form of the Hamiltonian is obtained using as a basis the set of Wannier states $w_{n}(z)$ for the test particle. For the case of a single impurity pinned at site $l$ we obtain

$$
\begin{aligned}
H_{l}= & \sum_{n}-J\left(\left|w_{n}\right\rangle\left\langle w_{n+1}|+| w_{n+1}\right\rangle\left\langle w_{n}\right|\right) \\
& -J^{d}\left(\left|w_{l}\right\rangle\left\langle w_{l \pm 1}|+| w_{l \pm 1}\right\rangle\left\langle w_{l}\right|\right)+u_{n-l}^{d d}\left|w_{n}\right\rangle\left\langle w_{n}\right| .
\end{aligned}
$$

In Eq. (7), beyond the standard nearest neighbor tunneling term $J$, we have included two terms due to the dipolar interaction: the first represents a nearest neighbor dipolar assisted hopping $J^{d}$, while the second contains the on-site energies $u_{n-l}^{d d}$ at site $n$. The Hamiltonian parameters can be calculated using the following expressions:

$$
\begin{aligned}
J & =-\int w_{n}^{*}(z)\left[-\frac{\hbar^{2}}{2 m} \frac{d^{2}}{d z^{2}}+s E_{R} \sin ^{2}(k z)\right] w_{n+1}(z) d z \\
J^{d} & =-\int w_{l}^{*}(z) w_{l+1}(z)\left|w_{l}^{(I)}\left(z^{\prime}\right)\right|^{2} U_{d d}^{1 D}\left(z-z^{\prime}\right) d z d z^{\prime} \\
u_{n-l}^{d d} & =\int\left|w_{n}(z)\right|^{2}\left|w_{l}^{(I)}\left(z^{\prime}\right)\right|^{2} U_{d d}^{1 D}\left(z-z^{\prime}\right) d z d z^{\prime} .
\end{aligned}
$$

The function $u_{n-l}^{d d}$ simply depends on the distance $|n-l|$ between the test particle and the impurity and provides the dipolar interaction between a single impurity and the test particle in the discretized formalism. 


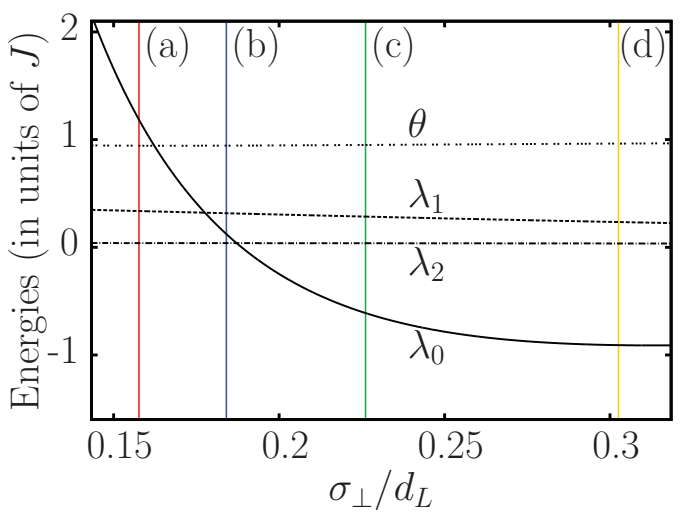

FIG. 2. (Color online) Site energies $\lambda_{0}, \lambda_{1}$, and $\lambda_{2}$, and hopping energy $\theta$ as function of $\sigma_{\perp}$ in units of $d_{L}$, for the case of dysprosium atoms with $D^{2} / d_{L}^{3}=0.016 E_{R}, \alpha=\pi / 2, \lambda_{L}=2 d_{L}=$ $570 \mathrm{~nm}, s_{(T)}=6$, and $s_{(I)}=30$. The colored vertical lines labeled by different letters correspond, respectively, to (a) $\sigma_{\perp} / d_{L}=0.158$, (b) $\sigma_{\perp} / d_{L}=0.184$, (c) $\sigma_{\perp} / d_{L}=0.226$, and (d) $\sigma_{\perp} / d_{L}=0.302$ and identify the set of parameters that we used for the calculation of the localization properties of the system.

In Fig. 2, we show the behavior of the quantities $\theta=$ $\left(J+J^{d}\right) / J$ and $\lambda_{n-l}=u_{n-l}^{d d} / J$ for $|n-l|=0,1$, and 2, for $D^{2} / d_{L}^{3}=0.016 E_{R}, \alpha=\pi / 2, s_{(T)}=6$, and $s_{(I)}=30$ as a function of $\sigma_{\perp}$ which is our control parameter. This value of $D^{2} / d_{L}^{3}$ corresponds to the case of dysprosium atoms trapped in an optical lattice generated by a laser of wavelength $\lambda_{L}=570 \mathrm{~nm} \mathrm{[30].}$

We note that, for this choice of parameters, we can reasonably set $\theta=1\left(J^{d}=0\right)$ and approximate the onsite energies for $|n-l| \geqslant 2$ by the asymptotic expression $\lambda_{n-l}=\lambda /|n-l|^{3}$ with $\lambda=D^{2} /\left(J d_{L}^{3}\right)$. They are, therefore, independent of the value of $\sigma_{\perp}$. Also $\lambda_{1}$ does not depend significantly on the radial confinement. Conversely the value of $\lambda_{0}$ strongly depends on $\sigma_{\perp}$ and can even vanish and become negative due to the anisotropy of the dipolar interaction.

In the presence of several impurities the different contributions have to be included summing over the occupied sites $\bar{l}$. Because of the impurity-impurity dipolar repulsion, we will impose that each impurity has to be preceded and followed by at least two empty sites. The resulting Hamiltonian,

$$
\begin{aligned}
H= & -J \sum_{n}\left(\left|w_{n}\right\rangle\left\langle w_{n+1}|+| w_{n+1}\right\rangle\left\langle w_{n}\right|\right) \\
& +\sum_{n} \varepsilon_{n}\left|w_{n}\right\rangle\left\langle w_{n}\right|,
\end{aligned}
$$

has hopping energies equal to $J$, and site energies

$$
\varepsilon_{n}=\sum_{\bar{l}} u_{n-\bar{l}}^{d d}=\sum_{l} \rho_{l} u_{n-l}^{d d} .
$$

The system is characterized by the properties of the impurity density distribution and the interaction potential. We introduce the notation $\langle\cdots\rangle$ to indicate the averaging for each lattice $l$ over different realizations of the disordered potential. Since, due to translational invariance such averages will not depend on the lattice site, the index $l$ will not appear in our notations for the correlation functions.
The discretized impurity density distribution $\rho_{l}$ is a stochastic variable with average value

$$
\left\langle\rho_{l}\right\rangle=\mathcal{C},
$$

corresponding to the impurity concentration $\mathcal{C}$, and density correlation function

$$
C_{\rho}(\ell)=\left\langle\rho_{l} \rho_{l+\ell}\right\rangle .
$$

The average value and the correlation functions of the full potential $\varepsilon_{l}$ can be extracted from the statistical properties of $\rho_{l}$ and the shape of the interaction potential. In particular, one can prove two important relations, first that the average value of the full potential is simply given by

$$
\left\langle\varepsilon_{l}\right\rangle=\mathcal{C} \sum_{n} u_{n}^{d d},
$$

which is the product of the impurity concentration and $\sum_{n} u_{n}^{d d}$. This last quantity can be thought of as a sort of spatial average of the interaction potential. Second, one can show that

$$
C_{\varepsilon}(\ell)=\left\langle\varepsilon_{l} \varepsilon_{l+\ell}\right\rangle=\sum_{j} C_{\rho}(\ell-j) C_{u}(j),
$$

namely, that the two-point correlation function is given by the convolution of the density correlation function $C_{\rho}(\ell)$ and

$$
C_{u}(\ell)=\sum_{n} u_{n}^{d d} u_{n+\ell}^{d d},
$$

which is the interaction potential correlation function.

For the case of a dipolar potential $\lim _{\ell \rightarrow \infty} C_{u}(\ell) \propto \ell^{-3}$, and for random impurities whose minimum distance is fixed to be three sites, as in our model, one has

$$
C_{\rho}(\ell)=\mathcal{C}^{2}+\left(\frac{\mathcal{C}}{1-2 \mathcal{C}}\right)^{\ell / 2}[A \cos (\kappa \ell)+B \sin (\kappa \ell)]
$$

with $\kappa=d_{L}^{-1}[\pi-\operatorname{atan} \sqrt{(4-9 \mathcal{C}) / \mathcal{C}}], \quad A=\mathcal{C}-\mathcal{C}^{2}$, and $B=$ $-\left[\sqrt{\mathcal{C}^{3}(1-2 \mathcal{C})}+\left(\mathcal{C}-\mathcal{C}^{2}\right) \cos \left(\kappa d_{L}\right)\right] / \sin \left(\kappa d_{L}\right)$.

Thus we can conclude that the impurity distribution introduces short-range correlations, while the shape of the interaction $u_{n}^{d d}$ is responsible for long-range correlations. The role and the competition between these two effects will be extensively discussed in Sec. IV.

For the full potential, let us also introduce the reduced correlation function, defined as

$$
c_{\varepsilon}(\ell)=\frac{\left\langle\varepsilon_{l} \varepsilon_{l+\ell}\right\rangle-\left\langle\varepsilon_{l}\right\rangle^{2}}{\left\langle\varepsilon_{l}^{2}\right\rangle-\left\langle\varepsilon_{l}\right\rangle^{2}}
$$

and the associated spectral density

$$
S(k)=\sum_{\ell} c_{\varepsilon}(\ell) e^{i k \ell} .
$$

In the following, we will use the square root of the variance of the full potential to quantify the potential strength

$$
W=\sqrt{\left\langle\varepsilon_{l}^{2}\right\rangle-\left\langle\varepsilon_{l}\right\rangle^{2}}
$$




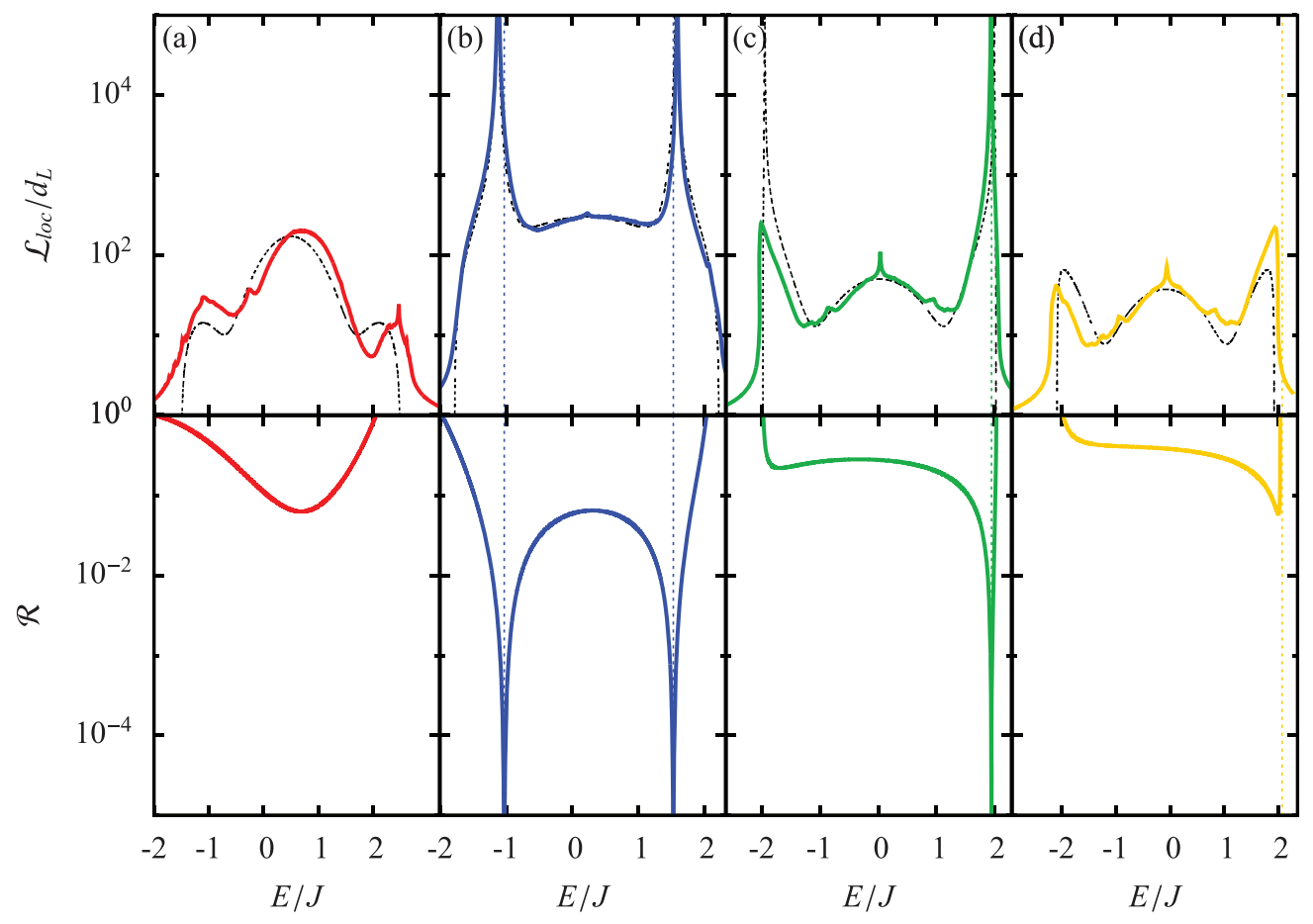

FIG. 3. (Color online) Top panel: localization length $\mathcal{L}_{\text {loc }}$ in units of the lattice spacing $d_{L}$ as a function of the energy in units of $J$ for $\mathcal{C}=1 / 4$, system sizes up to $10^{7}$ lattice sites, and averaged over 100 configurations. The black dashed lines correspond to the localization length calculated in the Born approximation $\mathcal{L}_{\text {loc }}^{(2)}$. Bottom panel: reflection coefficient $\mathcal{R}$ of the single impurity as a function of the energy in units of $J$. The vertical dashed lines indicate the energies for which the reflection coefficient vanishes $[\mathcal{R}(E)=0]$. From left to right, different plots refer to increasing values of $\sigma_{\perp}$, corresponding to the vertical lines in Fig. 2.

\section{NATURE OF THE SPECTRUM}

We study the nature of the spectrum of the test dipole by evaluating the Lyapunov exponent $\Lambda(E)$ through the asymptotic relation

$$
\Lambda(E)=\frac{1}{\mathcal{L}_{\mathrm{loc}}(E)}=\lim _{N \rightarrow \infty} \frac{1}{N d_{L}} \ln \left|\frac{G_{N, N}(E)}{G_{1, N}(E)}\right|,
$$

where $\mathcal{L}_{\text {loc }}(E)$ is the localization length, $G(E)=(E-H)^{-1}$ is the Green's function related to the Hamiltonian $H$ at energy $E$, and $G_{i, j}(E)=\langle i|G(E)| j\rangle$. The matrix elements $G_{1, N}(E)$ and $G_{N, N}$, where $N$ is the total number of lattice sites, have been computed by exploiting a renormalization-decimation scheme [38]. Our results, obtained as the average over several configurations, are shown in the upper row of Fig. 3, where we consider increasing values of $\sigma_{\perp}$, corresponding to the vertical lines in Fig. 2. We use the same color code in all figures and label corresponding simulations with the same letters $(a),(b)$, $(c)$, and $(d)$. Here and in the following, we consider system sizes up to $10^{7}$ lattice sites, fix $\mathcal{C}=1 / 4$, and average over 100 configurations. Each configuration is generated by randomly distributing the impurities along the lattice and forbidding those configurations where the minimum distance between impurities is less than three lattice sites.

As the value of $\sigma_{\perp}$ is increased, we observe very different localization regimes. Notably for certain values of $\sigma_{\perp}$, we observe divergences of the localization length, corresponding to the appearance of metallic states in the spectrum. This suggests the presence of delocalization effects induced by the correlations of the physical model under consideration. More precisely, for large positive values of $\lambda_{0}$, all states are clearly localized since the localization length is always finite [first panel $(a)$ ]. By increasing $\sigma_{\perp}$, for almost vanishing values of $\lambda_{0}$ the localization length exhibits two well defined peaks in two regions of the spectrum [second panel $(b)$ ]. Increasing $\sigma_{\perp}$ further, corresponding to negative values of $\lambda_{0}$, we observe the disappearance first of one of the two divergences [third panel $(c)$ ], and then of both of them [fourth panel $(d)$ ]. In this last panel no divergences of the localization length are observed, but there are still peaks at the band edges, that recall the diverging behavior previously observed.

The dashed black lines correspond to the localization length $\mathcal{L}_{\text {loc }}^{(2)}$ evaluated in the Born approximation, which corresponds to a second order perturbative calculation in the disorder strength [39-41],

$$
\Lambda^{(2)}(E)=\frac{1}{\mathcal{L}_{\mathrm{loc}}^{(2)}(E)}=\frac{W^{2}}{J^{2}} \frac{S(2 k(E))}{8 \sin ^{2}\left[k(E) d_{L}\right]},
$$

where the connection between $k(E)$ and the energy is given by the following relation $E=\left\langle\varepsilon_{n}\right\rangle+2 J \cos \left(k d_{L}\right)$. Let us note that the Born approximation gives, by construction, a symmetric localization length around the average value of the disorder $\left\langle\varepsilon_{n}\right\rangle$, since the spectral density $S(k)$ associated with $c(\ell)$ is always a symmetric function of $k$. Despite this fact, there is a noticeable agreement between the Born approximation and the exact numerical results, even in case (c) of a single divergence, where a strong peak at a negative energy is strongly reminiscent of the divergence found in the Born approximation. 


\section{ROLE OF CORRELATIONS}

With the aim of understanding why we are observing the appearance and the disappearance of metallic states in the spectrum by varying the radial confinement (and thus the effective 1D dipolar interaction), we analyze the effects of the short-range correlations introduced by the impurity density distribution and of the long-range correlations introduced by the dipolar potential between the test dipole and the dipolar impurities.

\section{A. Short-range correlations}

In order to isolate the role of short-range correlations in our model, we calculate the reflection coefficient for the case of a single impurity, as in Eq. (7), trapped at the site of index 0 of an infinite lattice. To get analytical results, we first neglect contributions beyond nearest neighbors. Therefore we assume that a single dipolar impurity modifies just a trimer of onsite energies $\left\{\lambda_{1}, \lambda_{0}, \lambda_{1}\right\}$, and we generally assume that it can modify also the hopping with nearest neighboring sites $\theta$.

A plane wave $\langle n \mid k\rangle=e^{i k n d_{L}}$, eigenstate of the unperturbed periodic Hamiltonian $H^{0}=\sum_{n=-\infty}^{+\infty}-J\left(\left|w_{n}\right\rangle\left\langle w_{n+1}\right|+\right.$ $\left.\left|w_{n+1}\right\rangle\left\langle w_{n}\right|\right)$ with energy $E=-2 J \cos \left(k d_{L}\right)$, is perturbed by the impurity and results in the following wave function

$$
\langle n \mid \varphi\rangle=\left\{\begin{array}{ll}
\tau e^{i k n d_{L}} & (n>1) \\
e^{i k n d_{L}}+r e^{-i k n d_{L}} & (n<-1)
\end{array},\right.
$$

where $\tau$ is the transmittance and $r$ the reflectance. Using the scattering formalism combined to the renormalizationdecimation scheme $[15,42,43]$, we obtain the following analytical formula for the reflection coefficient $\mathcal{R}=|r|^{2}$ of the single dipolar impurity:

$$
\mathcal{R}=\frac{\left\{\lambda_{1}\left(\frac{E}{J}\right)^{2}-\frac{E}{J}\left[1-\theta^{2}+\lambda_{1}^{2}+\lambda_{1} \lambda_{0}\right]-2 \theta^{2} \lambda_{1}+\lambda_{0}+\lambda_{1}^{2} \lambda_{0}\right\}^{2}}{\left\{\lambda_{1}\left(\frac{E}{J}\right)^{2}-\frac{E}{J}\left[1-\theta^{2}+\lambda_{1}^{2}+\lambda_{1} \lambda_{0}\right]-2 \theta^{2} \lambda_{1}+\lambda_{0}+\lambda_{1}^{2} \lambda_{0}\right\}^{2}+\theta^{4}\left[4-\left(\frac{E}{J}\right)^{2}\right]} .
$$

In the bottom row of Fig. 3, we plot $\mathcal{R}$ for the same parameters used for the calculation of the localization length, i.e., $\theta=1$ and $\lambda_{0}$ and $\lambda_{1}$ taken from the curves in Fig. 2. We observe that the calculation of the reflection coefficient of the single impurity provides a very good understanding of the behavior of the localization length: the energies where $\mathcal{R}$ tends to zero are exactly those where the localization length exhibits very large anomalous values. There is, therefore, a direct connection between the appearance of metallic states in the spectrum and the scattering properties of the single impurity. It has been previously shown by Dunlap et al. [14] that this kind of single impurity analysis can be used to interpret the transport properties of a system of $N$ lattice sites, where there are several randomly placed impurities. More precisely they proved that in such systems the number of single-particle states that show a metallic behavior, being extended over the full system, is of the order of $\sqrt{N}$. Notably this number of delocalized states is large enough to induce transport in the system, and initially localized wave packets show a superdiffusive spreading in the disordered potential. This means that this type of extended states are detectable in typical expansion experiments which can be performed with ultracold atomic gases [7,8].

It is remarkable that making use of the simple analytical expression (23) we can predict the localization properties of a rather complex system and the occurrence of metallic states in the spectrum. Studying the solutions of the equation

$$
\mathcal{R}(E)=0
$$

as a function of $\lambda_{0}$ and $\lambda_{1}$, one can extract the phase diagram in Fig. 4. We identify four different regions depending on the number of solutions of Eq. (24) and on their values. More precisely, if the solutions are both imaginary, no divergences are expected and all the states are exponentially localized [red region (a)]. If the solutions are real and inside the single impurity spectrum $E=-2 J \cos \left(k d_{L}\right)$, namely, when the roots satisfy the additional condition $|E|<2 J$, divergences are expected. Therefore, when the solutions are real, we can identify three additional scenarios: both solutions lie inside the spectrum [blue region (b)], only one solution lies inside the spectrum [green region (c)], and both solutions lie outside the spectrum [yellow region $(\mathrm{d})]$.

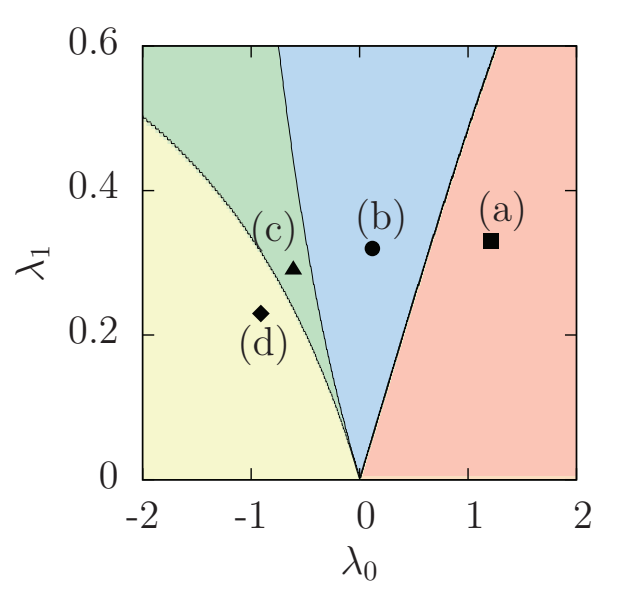

FIG. 4. (Color online) Phase diagram induced by short-range correlations extracted from the reflection coefficient in Eq. (23) for the single impurity case. The different regions correspond to different localization regimes obtained from the solution of Eq. (24). No real solutions of Eq. (24) correspond to the (a) red region. If Eq. (24) has real solutions, we can distinguish three cases depending on the number of solutions lying inside the spectrum: two solutions [(b) blue region], one solution [(c) green region], and no solutions [(d) yellow region]. The four markers in the diagram correspond to the simulations presented in Fig. 3 and to the values of $\sigma_{\perp}$ indicated by vertical lines in Fig. 2. 
In the diagram shown in Fig. 4, we identify with markers the values of $\lambda_{0}$ and $\lambda_{1}$ corresponding to the different plots of Fig. 3, where the dashed vertical lines mark the energies which verify the condition $\mathcal{R}(E)=0$. In particular the square in the red region (a) corresponds to the first plot in Fig. 3 where all states are localized; the circle in the blue region (b) corresponds to the second plot in Fig. 3, where we observe two resonances; the triangle in the green region (c) corresponds to the third plot in Fig. 3, where we observe one resonance; and the diamond in the yellow region (d) corresponds to the last plot in Fig. 3, where there are no resonances but the peak on the right shows a tendency to diverge due to the fact that the resonance lies just outside the single impurity spectrum.

Let us recall that, in our single impurity analysis, we considered the case where one isolated dipole induces just a trimer of site energies $\left\{\lambda_{1}, \lambda_{0}, \lambda_{1}\right\}$ and we neglected beyond nearest neighbor contributions. In other words, we neglected the effect of long-range correlations.

In the next subsection, we study in detail the role played by the dipolar tails that we neglected in this simplified calculation and we highlight the role played by long-range correlations.

\section{B. Long-range correlations}

In order to understand the role played by long-range correlations and place the dipolar case in a wider context, we investigate the localization properties of a disordered potential generated by an effective impurity-test particle interaction with tails decaying as $u_{|n|}^{\beta} \sim 1 /|n|^{\beta}$ where $\beta \geqslant 1$.

This is done by placing the impurities exactly as done in the dipolar case, keeping fixed the values of $\lambda_{0}$ and $\lambda_{1}$ and choosing $\lambda_{n}=u_{n}^{\beta} / J=\lambda /|n|^{\beta}$ for $n \geqslant 2$. The case $\beta=3$ recovers our physical model with dipolar interactions. Moreover, we shift and normalize the on-site energies in order to obtain the same average value $\left\langle\varepsilon_{n}\right\rangle$ and disorder strength $W$ that we had in the dipolar case. Following this procedure, we can really analyze the effect of long-range correlations keeping fixed the disorder strength $W$. In particular we considered values of $\beta$ ranging from 1 up to 5 and we also considered the case of $\beta=\infty$ that corresponds to $\lambda_{n}=0$ for $|n| \geqslant 2$.

The potential generated with this procedure has $C_{\rho}(\ell)$ which is unchanged and decays exponentially as previously discussed. This is due to the fact that the impurities are placed exactly in the same way as before. The correlation function associated with the interaction potential $C_{u}(\ell)$ is instead modified and using Eq. (15) one can show that it decays at large distances as $C_{u}(\ell) \sim 1 / \ell^{\beta}$ for $\beta>1$ and as $C_{u}(\ell) \sim \ln (\ell) / \ell$ for $\beta=1$. These asymptotic expressions determine the shape of the tails of the two-point correlation function $C_{\varepsilon}(\ell)$ of the random potential seen by the test dipole [see Eq. (14)] and consequently the reduced correlation function associated to the full potential $c_{\varepsilon}(\ell)$.

The effects played by long-range correlations are again studied by calculating numerically the localization length with the renormalization-decimation approach. In Fig. 5 we show the localization length $\mathcal{L}_{\text {loc }}$ calculated for different values of $\beta$. In particular we show a comparison between the two limiting cases of $\beta=\infty$ and $\beta=1$ and the physical case under consideration, i.e., the dipolar case $\beta=3$. We considered also

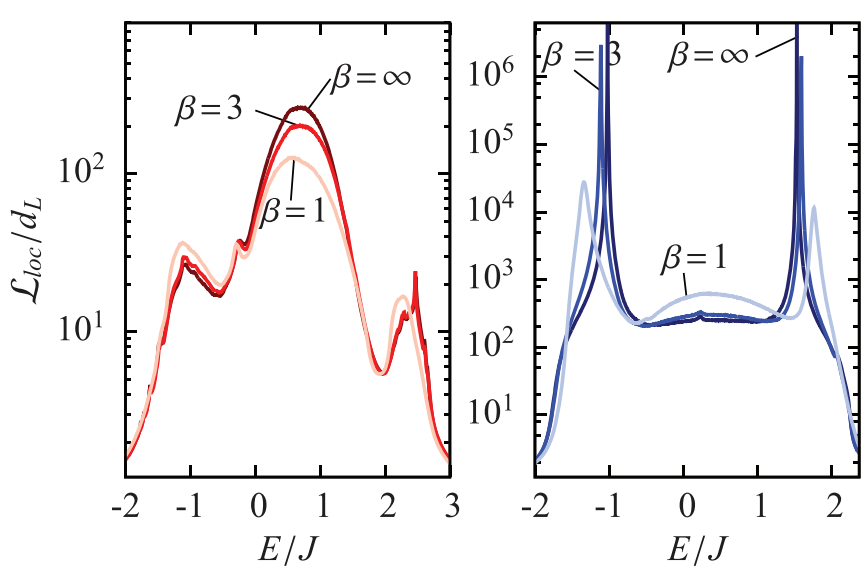

FIG. 5. (Color online) Localization length $\mathcal{L}_{\text {loc }}$ in units of the lattice spacing $d_{L}$ as a function of the energy in units of $J$ for $\beta=$ $1,3, \infty$, namely, different types of long-range correlations identified by the asymptotic decay of the tails of the two-point correlation function $C_{\varepsilon}(\ell)$. The left and right panels correspond to two different localization regimes induced by short-range correlations as shown in Figs. 3(a) and 3(b) for $\beta=3$ (see text for more details).

other values of $\beta$ but we do not show the results here since they are not particularly instructive. They just show an intermediate behavior between the two limiting cases reported here.

The two panels in Fig. 5 correspond to the two different sets of parameters already used for Figs. 3(a) and 3(b). In the left panel we show the case where the localization length is always finite, while in the right panel we show the case where there are two resonances in the spectrum. Therefore the two curves for $\beta=3$ are exactly the same curves which are shown in the upper rows of Figs. 3(a) and 3(b). We compare them with the case of the complete absence of long-range correlations ( $\beta=$ $\infty)$ and with the case of very slowly decaying correlations $(\beta=1)$.

In Fig. 5, there are two main features that we would like to stress here. The first is the effect on the divergencies in the localization length, discussed in the previous section. We observe that such divergencies tend to be beveled by long-range correlations. In fact for $\beta=\infty$, the localization length takes values of the order of the system size $\left(10^{7} d_{L}\right)$ signaling the presence of real metallic states in the system which extend over the full lattice. As the value of $\beta$ is reduced, corresponding to slower decaying tails, peaks in the localization length are still present but they are shifted towards the band edges and their height is decreased. This behavior is somehow expected, since the perfect resonance condition, obtained with the single impurity calculation presented in the previous section, is no more fulfilled in the presence of several impurities with overlapping slowly decaying tails. The tails tend to restore destructive interference in the forward direction and thus introduce localization in the system. However, from the results shown in Fig. 5, we can conclude that the effect of short-range correlations remains clearly visible also in presence of long-range correlations.

The second feature that we would like to highlight is the counterintuitive behavior of $\mathcal{L}_{\text {loc }}$ introduced by long-range correlations at the center of the band. In fact, depending on the set of parameters under consideration, long-range correlations 
have opposite effects on the localization length, which can either decrease (left panel), or be enhanced (right panel). This observation shows the highly nontrivial role played by long-range correlations in determining the localization properties of a disordered system and indicates a richer behavior with respect to what has been observed so far in the literature [22].

These features that we extracted from the numerical simulations reported in Fig. 5 are also captured within the Born approximation. We do not report the curves for $\mathcal{L}_{\text {loc }}^{(2)}$ calculated in the Born approximation, and just comment that the agreement between those curves and the exact numerical results is good, similar to that observed in Fig. 3.

One can understand the previous results based on the following properties: (i) in the Born approximation, $\Lambda^{(2)}$ is proportional to $S(2 k)$ and to $W^{2}$ which we have taken to be constant [see Eq. (21)]; (ii) the integral of $S(2 k)$ is a constant; (iii) the longer the range of the correlations, the larger $S(2 k)$ will be at the energy band edges. In the case of Fig. 5 (right), where for $\beta=\infty$, there are extended states, one can conjecture that, for decreasing $\beta$, property (iii) above together with the disappearance of the extended states lead to a decrease of $\Lambda^{(2)}$ at the energy band center, corresponding to an increase of the localization length, as observed. The case shown in Fig. 5 (left) cannot be explained based on similar simple arguments, since the increase of $S(2 k)$ at the energy band edges implies a nontrivial redistribution of the disorder spectral components all over the band. In the specific case, one observes an increase of $\mathcal{L}_{\text {loc }}$ at the energy band center, contrary to previous predictions [22].

Finally we would like to comment that we do not find the presence of mobility edges induced by long-range correlations as suggested in Refs. [23,44].

\section{CONCLUSIONS}

In this paper, we considered a set of dipolar impurities pinned at random positions in a deep optical lattice which create a disordered potential for an atom in a different internal state. An analysis of the statistical properties of the model showed that repulsive dipolar interactions between impurities introduce short-range correlations due to the fact that occupations of neighboring sites are forbidden.

The localization properties of the model were calculated by means of a renormalization-decimation technique which allowed us to calculate properties of very large systems and study the extended or localized nature of the states. We found that the presence of short-range correlations can give rise to different regimes. In particular, as the parameters of the system are changed, we observed regimes where one or more discrete sets of extended states appear in the spectrum. The occurrence of the different regimes can be predicted starting from an analytical expression obtained from the scattering of a single impurity.

Long-range correlations were studied not only for the dipolar case but also for a more general two-point correlation function decaying as $C(\ell) \sim 1 / \ell^{\beta}$, where the case $\beta=3$ corresponds to the dipolar case. We saw that long-range correlations in general tend to restore localization in the spectrum, but also lead to counterintuitive behaviors of the localization length. More precisely, depending on the regime under consideration, they can enhance or reduce localization at the center of the band.

Our work sheds light on the interplay between the role of short-range and long-range correlations and can be a guide for experiments devoted to the study of Anderson localization with ultracold dipolar gases. Natural extensions of the present work include the study of two-dimensional (2D) geometries and the role of interactions between many test dipoles.

\section{ACKNOWLEDGMENTS}

This work was supported by Grants No. CNRS-24543 and No. TUBITAK-210T050, by the LIA FSQL, by ERC through the QGBE grant, and by Provincia Autonoma di Trento. We are grateful to Franco Dalfovo, Iacopo Carusotto, Jean-François Schaff, and Luca Tessieri for useful discussions.
[1] P. W. Anderson, Phys. Rev. 109, 1492 (1958).

[2] P. W. Anderson, Philos. Mag. B 52, 505 (1985).

[3] H. Hu, A. Strybulevych, J. H. Page, S. E. Skipetrov, and B. A. van Tiggelen, Nat. Phys. 4, 945 (2008).

[4] D. Laurent, O. Legrand, P. Sebbah, C. Vanneste, and F. Mortessagne, Phys. Rev. Lett. 99, 253902 (2007).

[5] M. Störzer, P. Gross, C. M. Aegerter, and G. Maret, Phys. Rev. Lett. 96, 063904 (2006).

[6] M. Cutler and N. F. Mott, Phys. Rev. 181, 1336 (1969).

[7] J. Billy, V. Josse, Z. Zuo, A. Bernard, B. Hambrecht, P. Lugan, D. Clément, L. Sanchez-Palencia, P. Bouyer, and A. Aspect, Nature (London) 453, 891 (2008).

[8] G. Roati, C. D'Errico, L. Fallani, M. Fattori, C. Fort, M. Zaccanti, G. Modugno, M. Modugno, and M. Inguscio, Nature (London) 453, 895 (2008).

[9] S. S. Kondov, W. R. McGehee, J. J. Zirbel, and B. DeMarco, Science 334, 66 (2011).
[10] F. Jendrzejewski, A. Bernard, K. Mueller, P. Cheinet, V. Josse, M. Piraud, L. Pezzé, L. Sanchez-Palencia, A. Aspect, and P. Bouyer, Nat. Phys. 8, 398 (2012).

[11] F. L. Moore, J. C. Robinson, C. F. Bharucha, B. Sundaram, and M. G. Raizen, Phys. Rev. Lett. 75, 4598 (1995).

[12] J. Chabé, G. Lemarié, B. Grémaud, D. Delande, P. Szriftgiser, and J. C. Garreau, Phys. Rev. Lett. 101, 255702 (2008).

[13] E. Abrahams, P. W. Anderson, D. C. Licciardello, and T. V. Ramakrishnan, Phys. Rev. Lett. 42, 673 (1979).

[14] D. H. Dunlap, H.-L. Wu, and P. W. Phillips, Phys. Rev. Lett. 65, 88 (1990).

[15] J. F. Schaff, Z. Akdeniz, and P. Vignolo, Phys. Rev. A 81, 041604 (2010).

[16] T. A. Sedrakyan, J. P. Kestner, and S. Das Sarma, Phys. Rev. A 84, 053621 (2011).

[17] R. Farchioni, G. Grosso, and G. P. Parravicini, Phys. Rev. B 85, 165115 (2012). 
[18] E. Gurevich and O. Kenneth, Phys. Rev. A 79, 063617 (2009).

[19] P. Lugan, A. Aspect, L. Sanchez-Palencia, D. Delande, B. Grémaud, C. A. Müller, and C. Miniatura, Phys. Rev. A 80, 023605 (2009).

[20] L. Tessieri, J. Phys. A: Math. Gen. Phys. 35, 9585 (2002).

[21] U. Kuhl, F. M. Izrailev, and A. A. Krokhin, Phys. Rev. Lett. 100, 126402 (2008).

[22] A. Croy, P. Cain, and M. Schreiber, Eur. Phys. J. B 82, 107 (2011).

[23] F. A. B. F. de Moura and M. L. Lyra, Phys. Rev. Lett. 81, 3735 (1998).

[24] J. W. Kantelhardt, S. Russ, A. Bunde, S. Havlin, and I. Webman, Phys. Rev. Lett. 84, 198 (2000).

[25] F. A. B. F. de Moura and M. L. Lyra, Phys. Rev. Lett. 84, 199 (2000).

[26] T. Lahaye, C. Menotti, L. Santos, M. Lewenstein, and T. Pfau, Rep. Prog. Phys. 72, 126401 (2009).

[27] M. A. Baranov, M. Dalmonte, G. Pupillo, and P. Zoller, Chem. Rev. 112, 5012 (2012).

[28] A. Griesmaier, J. Werner, S. Hensler, J. Stuhler, and T. Pfau, Phys. Rev. Lett. 94, 160401 (2005).

[29] G. Bismut, B. Pasquiou, E. Maréchal, P. Pedri, L. Vernac, O. Gorceix, and B. Laburthe-Tolra, Phys. Rev. Lett. 105, 040404 (2010).

[30] M. Lu, N. Q. Burdick, S. H. Youn, and B. L. Lev, Phys. Rev. Lett. 107, 190401 (2011).
[31] K. Aikawa, A. Frisch, M. Mark, S. Baier, A. Rietzler, R. Grimm, and F. Ferlaino, Phys. Rev. Lett. 108, 210401 (2012).

[32] K. K. Ni, S. Ospelkaus, M. G. H. de Miranda, A. Peér, B. Neyenhuis, J. J. Zirbel, S. Kotochigova, P. S. Julienne, D. S. Jin, and J. Ye, Science 322, 231 (2008).

[33] C. H. Wu, J. W. Park, P. Ahmadi, S. Will, and M. W. Zwierlein, Phys. Rev. Lett. 109, 085301 (2012).

[34] P. Vignolo, Z. Akdeniz, and M. P. Tosi, J. Phys. B 36, 4535 (2003).

[35] U. Gavish and Y. Castin, Phys. Rev. Lett. 95, 020401 (2005).

[36] S. Sinha and L. Santos, Phys. Rev. Lett. 99, 140406 (2007).

[37] T. Koch, T. Lahaye, J. Metz, A. G. B. Fröhlich, and T. Pfau, Nat. Phys. 4, 218 (2008).

[38] R. Farchioni, G. Grosso, and G. Pastori Parravicini, Phys. Rev. B 45, 6383 (1992).

[39] J. M. Luck, Phys. Rev. B 39, 5834 (1989).

[40] F. M. Izrailev and A. A. Krokhin, Phys. Rev. Lett. 82, 4062 (1999).

[41] F. Izrailev, A. Krokhin, and N. Makarov, Phys. Rep. 512, 125 (2012).

[42] R. Farchioni, P. Vignolo, and G. Grosso, Phys. Rev. B 60, 15705 (1999).

[43] M. R. Bakhtiari, P. Vignolo, and M. P. Tosi, Physica E 28, 385 (2005).

[44] A. M. García-García and E. Cuevas, Phys. Rev. B 79, 073104 (2009). 